\title{
Doravirine and Its Potential in the Treatment of HIV: An Evidence-Based Review of the Emerging
} Data

\author{
Alexander E Rock (iD) \\ Jeremy Lerner ${ }^{2}$ \\ Melissa E Badowski (iD) \\ 'University of Illinois, College of \\ Pharmacy, Department of Pharmacy \\ Practice, Section of Infectious Diseases \\ Pharmacotherapy, Chicago, IL 606/2, \\ USA; ${ }^{2}$ University of Illinois, College of \\ Pharmacy, Department of Pharmacy \\ Practice, Chicago, IL 606/2, USA
}

This article was published in the following Dove Press journal: HIVIAIDS - Research and Palliative Care

\begin{abstract}
The utility of doravirine in the management of HIV-1 infection is approved for use in patients who are antiretroviral-naïve as well as patients who have achieved stable virologic suppression and are interested in replacing their current antiretroviral therapy. The role of doravirine continues to evolve as data emerges on the potential for new combination therapy with the investigational agent, islatravir, as well as a potential strategy to minimize post-marketing safety concerns with recommended first-line agents, such as integrase inhibitors. The goal of this review is to assess recent and emerging data on the non-nucleoside reverse transcriptase inhibitor, doravirine.
\end{abstract}

Keywords: doravirine, HIV-1, antiretroviral therapy, non-nucleoside reverse transcriptase inhibitor

\section{Introduction}

Doravirine, the most recent non-nucleoside reverse transcriptase inhibitor (NNRTI), is available as a co-formulated single-tablet regimen (STR) including lamivudine and tenofovir disoproxil fumarate (Delstrigo ${ }^{\mathrm{TM}}$ ) and independently distributed as Pifeltro $^{\mathrm{TM}}$. Doravirine is approved as initial therapy as well as for those virologically suppressed on a stable antiretroviral (ARV) regimen. ${ }^{1}$ This addition provides much needed variety to the NNRTI class with a daily medication that has less frequent side effects compared to other NNRTIs, maintains activity in the presence of common NNRTI resistance mutations (ie K103N, E138K, Y181C, etc.) and potentially provides a therapeutic alternative for individuals experiencing adverse events from other medication classes (ie integrase strand transfer inhibitors (INSTIs), protease inhibitors (PIs), etc.). Currently, the European AIDS Clinical Society (EACS) recommends the use of doravirine/lamivudine/tenofovir disoproxil fumarate STR for initial therapy in patients who are ARV-naïve whereas the United States Department of Health and Human Services (DHHS) reserves this combination for certain clinical situations. ${ }^{2,3}$

With emerging data suggesting possible links to weight gain with both tenofovir alafenamide and the INSTI class there is a need for highly effective antiretroviral therapy (ART) with benign side effect profiles. ${ }^{4-6}$ To date, there is minimal data addressing weight gain with doravirine. Recent cumulative data from three clinical trials (Phase 2, P007; Phase 3, DRIVE-FORWARD and DRIVE-AHEAD) concluded there may be slightly more weight gain associated 


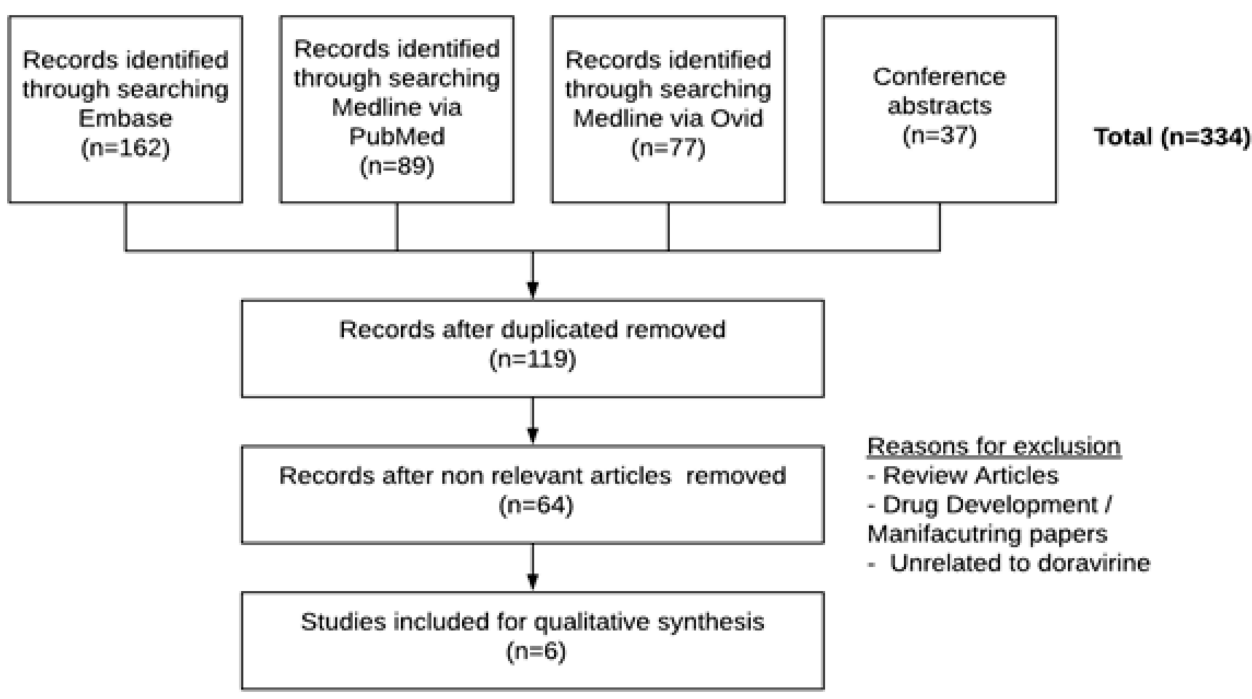

Figure I PRISMA flow diagram for search methodology.

Note: Adapted from Moher D, Liberati A, Tetzlaff J, Altman DG, The PRISMA Group (2009). Preferred Reporting Items for Systematic Reviews and Meta-Analyses: The PRISMA Statement. PLoS Med 6(7): el000097. Creative Commons license and disclaimer available from: http://creativecommons.org/licenses/by/4.0/legalcode. ${ }^{36}$

with doravirine than efavirenz but no significant weight gain when compared to boosted darunavir regimens in treatment-naïve people living with HIV (PLWH). ${ }^{7}$ The co-formulation of doravirine with lamivudine and tenofovir disoproxil fumarate may become more appealing because this STR may be an ideal alternative for patients suffering from ongoing issues related to weight gain or hypertriglyceridemia. One potential concern with the coformulation of tenofovir disoproxil fumarate focuses on renal and bone health. ${ }^{8}$ Tenofovir disoproxil fumarate has a significant history associated with increased rates of renal injury, and decreased bone mineral density. Although these events are serious, fortunately their incidence is rare.

When highly treatment-experienced patients are unable to achieve virologic suppression it is not an uncommon practice to manage someone an INSTI with a boosted PI with or without a NRTI backbone, based on resistance patterns and tolerability. In circumstances where this boosted PI needs to be avoided, such as patients who are awaiting transplantation or individuals with drug interactions precluding the use of a boosted PI, doravirine becomes an attractive option. Although this occurs in clinical practice there has yet to be a randomized clinical trial performed to validate this approach.

Another potential role of doravirine is being evaluated in combination with lamivudine and islatravir, ${ }^{9}$ a nucleoside reverse transcriptase translocation inhibitor (NRTTI). With renewed interest in doravirine, the goal of this manuscript is to review current and emerging data on the role of doravirine in the management of HIV-1 infection.

\section{Methodology}

A literature search current through 27 March 2020 was conducted using Embase $(\mathrm{n}=162)$, Medline via PubMed $(n=89)$, and Medline via Ovid $(n=77)$ with the search terms utilized being doravirine OR mk-1439. The same terms were used to review abstract results from the Conference on Retroviruses and Opportunistic Infections (CROI) $(n=13)$, International AIDS Society Conference on HIV Science (IAS) $(n=6)$, European AIDS Clinical Society (EACS) $(n=7)$, International Workshop of Clinical Pharmacology on HIV and Hepatitis Therapy $(n=5)$, and IDWeek $(n=6)$. Searches of these databases were limited to the English language and adult patients but were not limited beyond these criteria. The above results were compared for duplicates and resulted in $\mathrm{n}=119$ unique published papers and $n=37$ conference abstracts (Figure 1 ).

\section{Results}

The greatest amount of data existing on doravirine is derived from the DRIVE trials along with Phase II dose finding trials (Table 1). ${ }^{9-17}$ These trials outline efficacy and safety data for doravirine and are the basis for its approval by the US Food and Drug Administration. Longterm efficacy data is available up to 96 weeks in the DRIVE-FORWARD ${ }^{10,11}$ and DRIVE-AHEAD ${ }^{14,15}$ trials. These studies assessed treatment-naïve patients as well as 


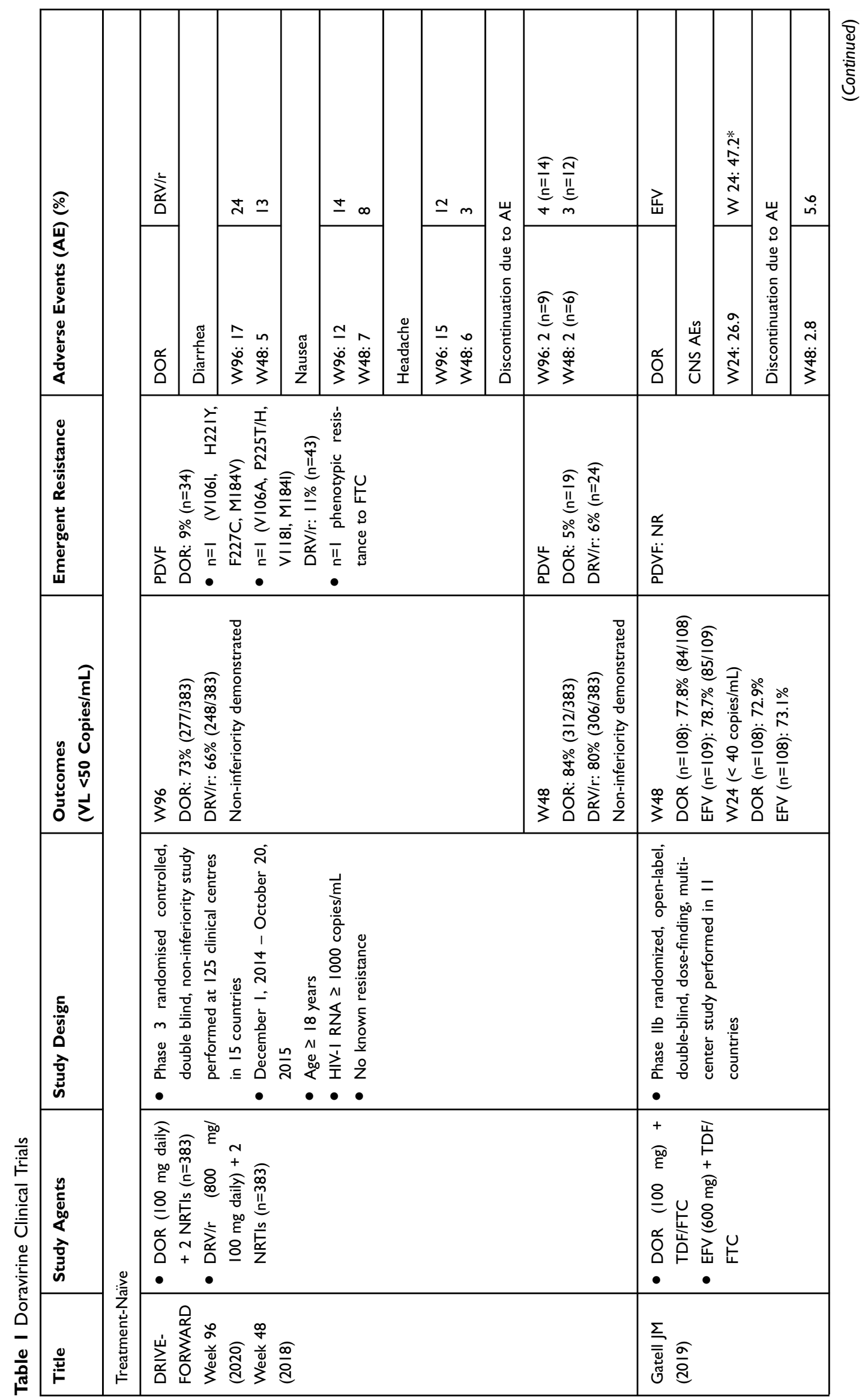




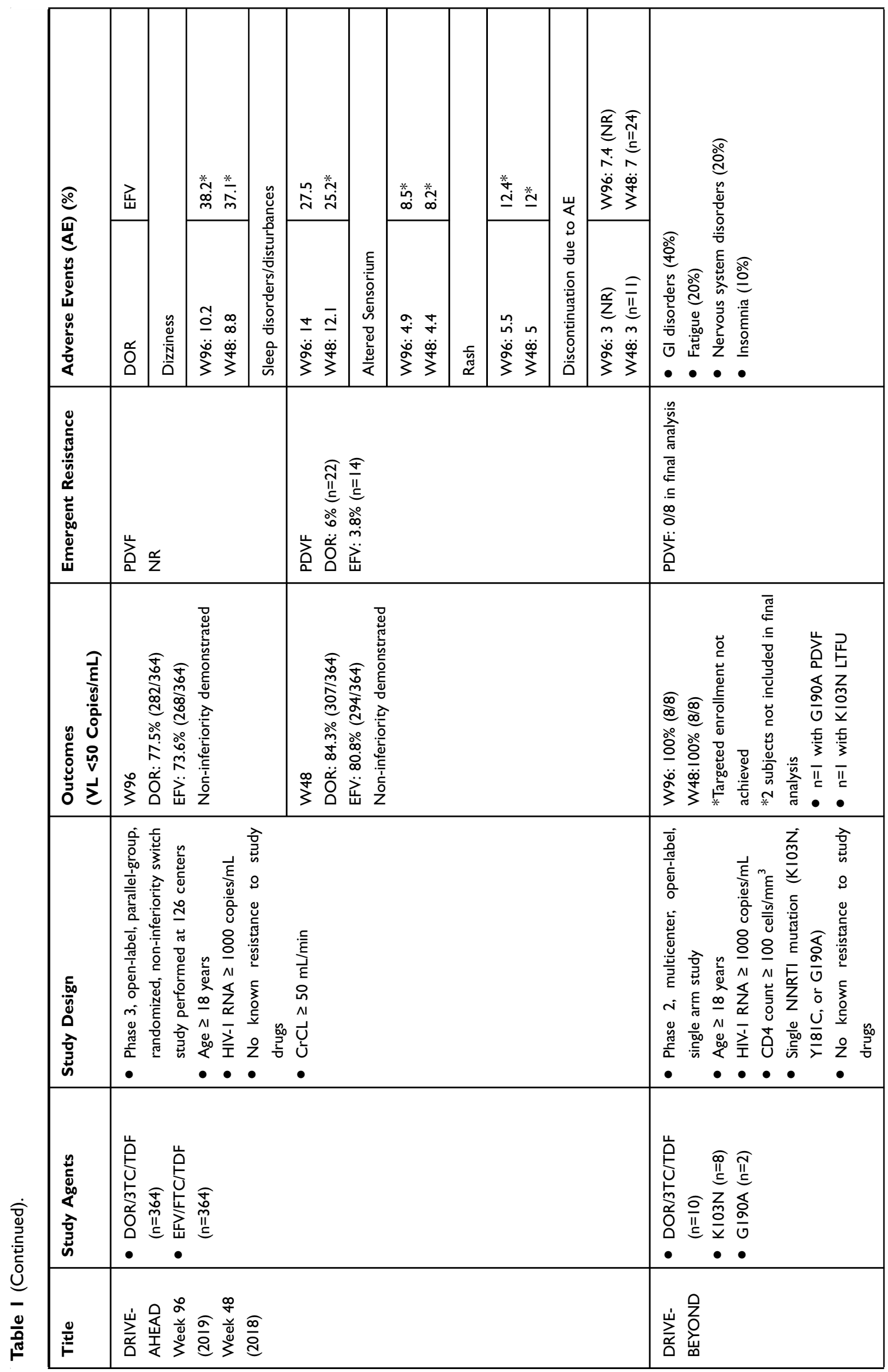




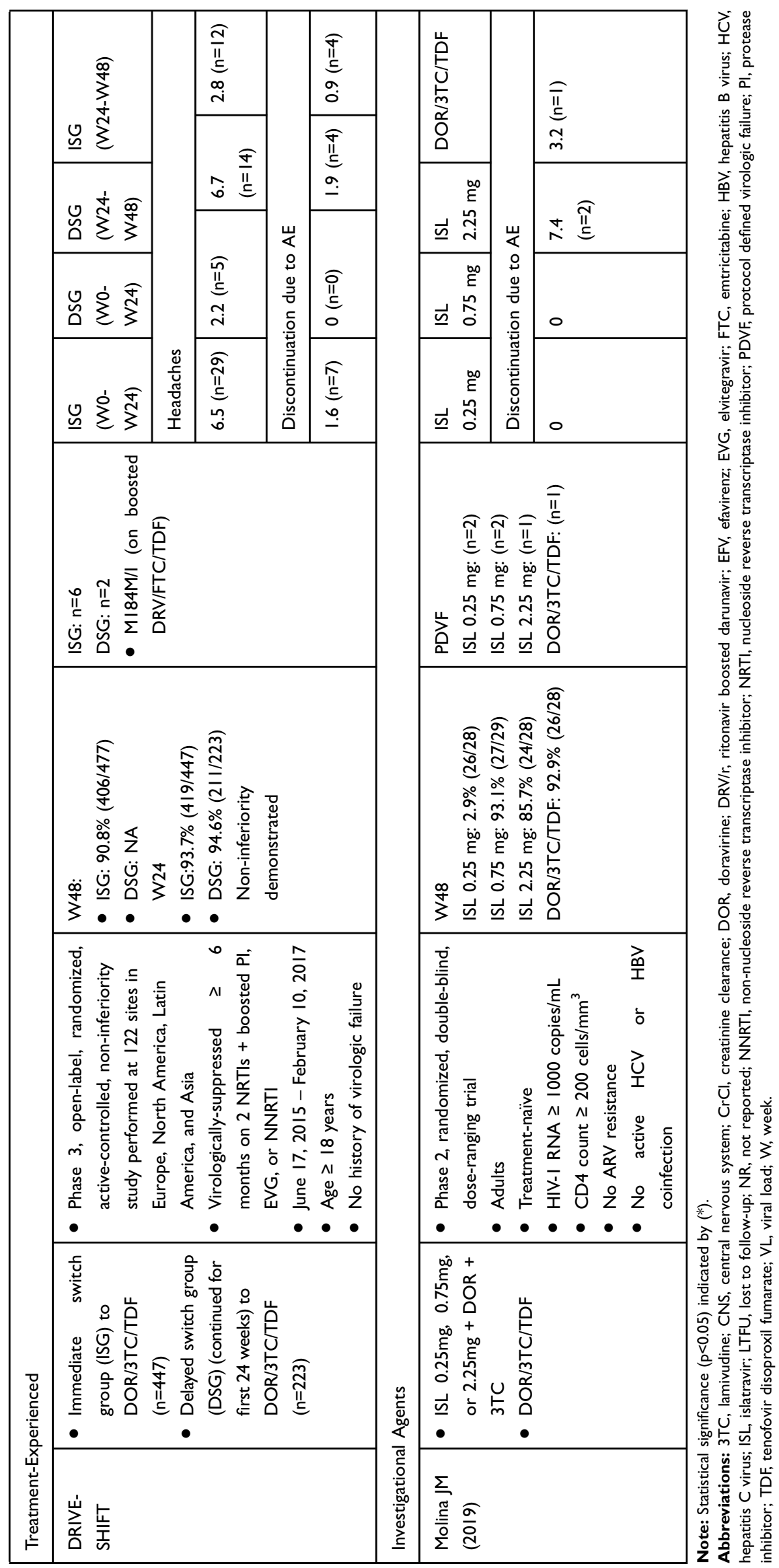


those switching from stable ART. The DRIVE trials in treatment-naïve only directly compare doravirine to efavirenz/emtricitabine/tenofovir disoproxil fumarate and boosted darunavir. The DRIVE-Shift trial did include patients that switched to a doravirine regimen from either a boosted PI, elvitegravir, or NNRTI-based regimen, however, it did not stratify the results based on these characteristics. ${ }^{17}$ Across all of the DRIVE studies there was a consistent trend of doravirine being better tolerated than its comparator, the only exception being the DRIVEFORWARD trial where the incidence of headache was higher at both 48 and 96 weeks for the doravirine arm compared to boosted darunavir. ${ }^{10,11}$

DRIVE-FORWARD was a phase 3, double blind, noninferiority trial, comparing treatment-naïve patients whom were started in a 1:1 ratio on either doravirine or darunavir boosted with ritonavir with investigator selected NRTI backbone therapy. The mean effect on low-density lipoprotein (LDL-C), non-high density lipoprotein (HDL-C), HDL, total cholesterol (TC), and triglycerides (TG) at 96 weeks was $(-0.4 \mathrm{mg} / \mathrm{dL},-0.5 \mathrm{mg} / \mathrm{dL}, 4.5 \mathrm{mg} / \mathrm{dL}, 4.1 \mathrm{mg} /$ $\mathrm{dL}$, and $-1.1 \mathrm{mg} / \mathrm{dL})$ and (14.0 $\mathrm{mg} / \mathrm{dL}, 17.7 \mathrm{mg} / \mathrm{dL}$, $4.2 \mathrm{mg} / \mathrm{dL}, 21.9 \mathrm{mg} / \mathrm{dL}$, and $22.5 \mathrm{mg} / \mathrm{dL}$ ), for doravirine and darunavir boosted with ritonavir regimens, respectively. A significant difference was observed with a $95 \%$ confidence interval (CI) for all parameters of the boosted darunavir arm as well as HDL and cholesterol in the doravirine arm. ${ }^{10,11}$

DRIVE-AHEAD was a phase 3, double blind, noninferiority trial comparing treatment-naïve patients whom were started in a 1:1 ratio on either doravirine/lamivudine/ tenofovir disoproxil fumarate or efavirenz/emtricitabine/ tenofovir disoproxil fumarate. At 0,48 , and 96 weeks, measured lipids were associated with a positive effect in the doravirine group. The mean effect on LDL and nonHDL at 96 weeks was $(-0.62 \mathrm{mg} / \mathrm{dL}$ and $-2.14 \mathrm{mg} / \mathrm{dL})$ and $(10.78 \mathrm{mg} / \mathrm{dL}$ and $14.95 \mathrm{mg} / \mathrm{dL})$ for the doravirine and efavirenz arm, respectively. Fasting LDL-C and non-HDL $-\mathrm{C}$ increased in the efavirenz group but not in the doravirine group, yet the change in total cholesterol/HDL-C ratio was similar. $^{14,15}$

DRIVE-SHIFT was a switch study assessing two groups (baseline regimen vs doravirine/lamivudine/tenofovir disoproxil fumarate) before and after a switch where all patients ultimately received doravirine/lamivudine/tenofovir disoproxil fumarate. No weight gain or abnormal laboratory results related to changes in lipid profiles led to discontinuation of therapy. This study demonstrated superior lipid profiles for doravirine/lamivudine/tenofovir disoproxil fumarate as compared with boosted PI therapy. Statistically significant differences in both LDL and nonHDL at week 24 ( $p<0.0001)$ were observed. However, the population effect of this may be limited, as only $1.6 \%$ of patients $(n=3 / 184)$ in the baseline regimen group had a grade 3 laboratory abnormality value or greater (defined as $\mathrm{LDL} \geq 190 \mathrm{mg} / \mathrm{dL}$ ) at the end of the 24 -week pre-switch time period. These three individuals did return to $<190 \mathrm{mg} / \mathrm{dL}$ after 24 weeks on doravirine/lamivudine/ tenofovir disoproxil fumarate. ${ }^{17}$

Taken together, the DRIVE trials demonstrate that doravirine has proven efficacy over long periods of time while maintaining an acceptable adverse effect profile that may distinguish it from other regimens. One limitation noticed across all of the DRIVE trials is that the majority of participants were male, and there was a lack of data representing women who were pregnant.

\section{Emerging Literature and Discussion Resistance}

Additional doravirine resistance data is beginning to emerge. One analysis evaluated 9764 sequences of ARVnaïve samples in France, Greece, and Italy for the development of doravirine resistance-associated mutations (RAM). At least one doravirine RAM was identified in $1.4 \%$ ( $\mathrm{n}=$ 137) of this population with most frequent mutations being V108I $(\mathrm{n}=62 ; 0.6 \%)$ Y188L $(\mathrm{n}=18 ; 0.2 \%), \mathrm{H} 221 \mathrm{Y}(\mathrm{n}=$ $18 ; 0.2 \%)$ and $\mathrm{Y} 318 \mathrm{~F}(\mathrm{n}=23 ; 0.2 \%) .{ }^{18}$ However, it is important to keep in mind that these tests were performed prior to doravirine being widely available and the mutations associated with doravirine resistance may not have been identified at the time. Additionally, this was only a genotypic assessment and does not demonstrate how all of these viruses would have phenotypically responded to doravirine. The same group went on to assess 9,199 sequences of which 381 belonged to treatmentexperienced NNRTI failing patients. This subset, according to the Stanford algorithm, demonstrated a $42 \% \quad(n=160)$ prevalence of doravirine resistance. ${ }^{19}$

An algorithm to predict transmitted drug resistance (TDR) in treatment-naïve patients in Spain demonstrated infrequent TDR associated with doravirine (1.8\%) and PIs $(0.8 \%)$ compared to other NNRTIs $(13.5 \%)$ and NRTIs $(3.0 \%)$. Unfortunately, TDR to INSTIs was not assessed in this analysis. ${ }^{20}$ Another study evaluated 6,893 NNRTIexperienced patients in Italy and identified intermediate 
doravirine resistance (ie V106A) in $12.7 \%$ and high-level doravirine resistance in $6.1 \%$ with the most common mutation being Y188L. Previous treatment with efavirenz or etravirine was associated with high-level doravirine resistance while rilpivirine was associated with a lower probability of high-level doravirine resistance. ${ }^{21}$ A study conducted by Ayitewala et al, assessed Ugandan patients between 2006 and 2017 who failed therapy after a year of efavirenz-based treatment and found 78.5\% $(n=143)$ of these patients demonstrated high levels of resistance to efavirenz. When this group was analyzed for crossresistance to the other NNRTIs, only $25.2 \%(n=36)$ were susceptible to etravirine and rilpivirine and $15.4 \%(\mathrm{n}=22)$ were susceptible to doravirine. ${ }^{22}$

\section{Pharmacokinetic/Pharmacodynamic}

An excellent review performed by Boyle and colleagues addresses the general pharmacodynamic, pharmacokinetic, and drug interaction profile of doravirine. ${ }^{23}$ Since its publication in August 2019, a phase one study evaluated oral granules as an alternative formulation for the single tablet of combination doravirine/tenofovir disoproxil fumarate/ lamivudine. Equivalence of each component administered was evaluated as granules as compared to their tablet equivalent when taken alone and in combination with either apple sauce or vanilla pudding. The absorption of this formulation was determined to be equivalent to oral tablets. Although this formulation is not currently available, it would be an important option for patients unable to tolerate or take oral tablets. ${ }^{24}$

Another study assessed serum doravirine concentrations after 72 hours of treatment cessation. This study, importantly, demonstrated that at 72 hours after a single dose of doravirine, $100 \%$ of the participants had an average trough concentration of $39 \mathrm{ng} / \mathrm{mL}$ (range: 33-61 ng/ $\mathrm{mL}$ ) which was above the goal of $23 \mathrm{ng} / \mathrm{mL}$ set during drug development. This is significant because it demonstrates the forgiveness that standard doravirine dosing would have in response to occasional non-compliance. ${ }^{25}$

\section{Investigational Formulations}

Another emerging role for the use of doravirine is being evaluated with the use of the NRTTI, islatravir. In a dose finding study (Table 1), various doses of islatravir were paired with lamivudine and doravirine. Participants who achieved viral suppression ( $<50$ copies/mL) at week 20 or later stopped lamivudine at their next visit and continued with doravirine and islatravir. ${ }^{9}$ No differences in weight change or body mass index were observed from baseline and were similar between the varying doses of islatravir combined with doravirine and doravirine/lamivudine/tenofovir disoproxil fumarate. A significantly lower impact was observed on hip bone mineral density (BMD) with islatravir combined with doravirine when compared to doravirine/lamivudine/tenofovir disoproxil fumarate yet changes in spine BMD were similar between the groups. $^{26}$ The two-drug combination of doravirine $100 \mathrm{mg}$ and islatravir $0.75 \mathrm{mg}$ is being considered in treatment-naïve, virologically suppressed, and highly treatment-experienced populations. ${ }^{27}$

\section{Clinical Implications for Doravirine}

The role of doravirine continues to evolve in clinical practice as new data and potential combinations emerge along with concerns of post-marketing safety data associated with other drug classes. Although doravirine is approved as initial therapy in patients who are ARVnaïve, it is currently recommended to obtain a genotype prior to the initiation of ART. In patients who are treatment-naïve and no TDR is identified, the STR of doravirine/lamivudine/tenofovir disoproxil fumarate may be an appropriate option since baseline resistance to doravirine is rather low but baseline genotypes must also consider the presence of NRTI mutations. The development of doravirine RAMs while on therapy is another concern in the setting of medication non-adherence along with previous use of efavirenz and etravirine. ${ }^{21}$ Although doravirine may have a higher genetic barrier to resistance compared to first-generation NNRTIs, second-generation INSTIs (dolutegravir and bictegravir) and PIs are more forgiving in settings of medication non-adherence and are associated with higher genetic barriers to resistance. Furthermore, development of $\mathrm{V} 106 \mathrm{~A} / \mathrm{M}$ or $\mathrm{Y} 188 \mathrm{~L}$ with the use of doravirine can precipitate the development of crossresistance across the NNRTI class. ${ }^{10,28}$

In patients experiencing side effects from other ARVs, such as weight gain with INSTIs or with tenofovir alafenamide, the role of doravirine may emerge as a potential solution to reduce weight gain as well as long-term metabolic consequences. Although not directly compared to INSTIs, data on the effect of weight gain with doravirine use was only recently presented. While no difference in weight gain from baseline was observed between doravirine $(\mathrm{W} 48=1 \mathrm{~kg}$; W96 = $1.5 \mathrm{~kg})$ and boosted darunavir $(\mathrm{W} 48=0.59 \mathrm{~kg} ; \mathrm{W} 96=0.7 \mathrm{~kg})$, a significant difference was observed between doravirine and efavirenz (W48 = 
$0 \mathrm{~kg}$; W96 $=1 \mathrm{~kg})$ based on $48(\mathrm{p}<0.001)$ and 96 $(\mathrm{p}=0.020)$ week data. ${ }^{7}$ In addition, DRIVE-AHEAD ${ }^{14,15}$ and DRIVE-FORWARD ${ }^{10,11}$ demonstrated better lipid profiles when doravirine was compared to efavirenz or boosted-darunavir. Additionally, DRIVE-SHIFT also demonstrated better lipid outcomes associated with doravirine, data on baseline regimens (ie boosted darunavir or boosted elvitegravir) were not separated to assess the degree of difference. ${ }^{17}$ A main limitation in this study is the lack of a true comparison to recommended INSTIs, dolutegravir and bictegravir.

When evaluating doravirine for use in renal dysfunction, if administered as an individual agent, dosage adjustments are not required regardless of creatinine clearance. ${ }^{1}$ Yet, a major concern with co-formulating doravirine with lamivudine and tenofovir disoproxil fumarate is renal and bone safety. Although bone and renal parameters were not specifically assessed in the DRIVE trials, 96-week data obtained from the DRIVE-FORWARD study was not associated with significant differences between Grade 2 or 3 adverse events when comparing doravirine or ritonavir-boosted darunavir with investigator selected NRTI backbone (tenofovir disoproxil fumarate/emtricitabine or abacavir/lamivudine). Unfortunately, data comparing backbone therapy was not described in order to more clearly evaluate changes in kidney function. ${ }^{10,11}$ The DRIVE-AHEAD study also did not specifically evaluate bone and renal parameters but did note fractures and drug-related adverse events occurred in less than $1 \%$ of the treatment group. Grade 3 adverse events related to changes in creatine were identified in $1.9 \%$ of the doravirine/lamivudine/tenofovir disoproxil fumarate group and $0.8 \%$ of the efavirenz/emtricitabine/tenofovir disoproxil fumarate group [95\% CI $1.1(-0.7,3.2)]$ based on 48-week supplementary data. ${ }^{14,15}$ Updated 96-week data demonstrated a rise in serum creatinine by greater than 1.3-1.8 $\mathrm{x}$ Upper Limit of Normal (ULN) or increase of greater than $0.3 \mathrm{mg} / \mathrm{dL}$ above baseline (doravirine $=3 \%$; efavirenz $=$ $2 \%$ ) or increase of $\geq 1.5 \times$ above baseline (doravirine $=3 \%$; efavirenz $=2 \%$ ) was not significantly different between groups. ${ }^{29}$ Finally, of 670 patients enrolled in the DRIVESHIFT study, only 2 individuals experienced moderate $(n=1)$ or serious $(n=1)$ adverse renal events which resolved upon cessation of doravirine-based therapy. Post-marketing data should continue to evaluate the development of adverse renal effects in patients taking doravirine/lamivudine/tenofovir disoproxil fumarate.

One potential advantage of co-formulating doravirine with tenofovir disoproxil fumarate as opposed to tenofovir alafenamide may be the concern for alopecia which was recently described in a case series of 6 African American women. Five out of 6 women reported improvement of alopecia 1-5 months upon cessation of tenofovir alafenamide therapy. Although this cause-effect relationship is in a rather small number of individuals, additional studies should evaluate this potential adverse effect in greater detail. $^{30}$

Lack of food requirements make doravirine an attractive option when compared to rilpivirine, etravirine, efavirenz, PIs, and elvitegravir/cobicistat. In addition, while the DRIVE trials give a baseline level of efficacy, other evidence has shown that doravirine can be an effective NNRTI that has a place in modern HIV therapy. A common reason to switch between therapies has often been due to drug interactions. The lack of certain drug interactions with doravirine can potentially distinguish it from other therapies. For example, doravirine was not associated with any clinically significant interactions between pantoprazole or an aluminum-magnesium based antacid. ${ }^{31}$ Although the true number of patients living with HIV and requiring antacid therapy is not well defined, one study distributed a validated gastroesophageal reflux disease (GERD) questionnaire between two HIV clinics in Canada to 120 patients to assess the presence of GERD. Eighty-five patients were included in the analysis where 68 (81\%) met criteria for diagnosis of GERD and 65 (95.6\%) were taking medications, the majority being histamine 2 receptor antagonists (H2RA), to alleviate symptoms. ${ }^{32}$ While doravirine does not interact with H2RA or proton pump inhibitors, rilpivirine and atazanavir do have significant interactions.

Another common drug interaction to be concerned with is polyvalent cations and the widely used INSTIs. A retrospective analysis performed in Delaware, USA, identified 152 (42\%) clinic patients as receiving a polyvalent cation and an INSTI. Forty-six patients (13\%) went on to develop virologic failure with the use of either dolutegravir (15\%), elvitegravir $(11 \%)$, or raltegravir (18\%), carrying an average 2.3 times risk for virologic failure when compared to those not reporting polyvalent cation use [95\% CI $1.2-4.4]{ }^{33}$ These interaction profiles can potentially distinguish doravirine from other currently available agents.

An important drug interaction that warrants discussion is between doravirine and the rifamycin drug class. While the administration of rifampin in combination with doravirine is not recommended, as concentrations of doravirine 
will be substantially decreased, rifabutin can be considered as long as doravirine is dosed twice daily. ${ }^{34,35}$

While the ideal candidate for doravirine STR must have a creatinine clearance of $\geq 50 \mathrm{~mL} / \mathrm{min}$, it can be administered irrespective of CD4 count or HIV-1 viral load. Doravirine, as a single agent, can be administered regardless of creatinine clearance and may be beneficial in patients who have susceptible virus to doravirine but documented resistance to other ARVs. Caution should be observed in patients with underlying NNRTI exposure and resistance since the durability of doravirine may be compromised. Both formulations of doravirine may be considered for patients experiencing side effects or drug interactions to other ARVs. While the role of doravirine continues to evolve, the focus of future studies should center on longterm safety and efficacy compared to current recommended regimens and standard of care as well as the potential combination with new agents being evaluated.

\section{Disclosure}

The authors report no conflicts of interest in this work.

\section{References}

1. Pifeltro [package insert]. Whitehouse Station, NJ: Merck \& CO., Inc; 2019.

2. European AIDS Clinical Society. EACS Guidelines version 10.0. Available from: https://www.eacsociety.org/files/2019_guidelines-10. 0 final.pdf. Accessed March 25, 2020.

3. U. S. Department of Health and Human Services. Guidelines for the use of antiretroviral agents in adults and adolescents with HIV Available from: https://files.aidsinfo.nih.gov/contentfiles/lvguide lines/AA_Tables.pdf. Accessed March 25, 2020.

4. Ruane PJ, Clarke A, Post FA, et al. Phase 3 randomized, controlled DISCOVER study of daily emtricitabine/tenofovir alafenamide (F/TAF) or emtricitabine/tenofovir disoproxil fumarate (F/TDF) for HIV pre-exposure prophylaxis (PrEP): week 96 results. In: 17th Annual European AIDS Clinical Society (EACS); November 6-9, 2019; Basel, Switzerland.

5. Venter WDF, Moorhouse M, Sokhela S, et al. Dolutegravir plus two different prodrugs of tenofovir to treat HIV. $N$ Engl J Med. 2019;381 (9):803-815. doi:10.1056/NEJMoa1902824

6. Perez SE, Chow SP, Kania A, Goldberg R, Badowski ME. Weighing in on the role of Integrase Strand Transfer Inhibitors (INSTIs) on weight gain: fact or fiction? Current Infectious Disease Reports [Accepted].

7. Orkin C. Effect of doravirine on body weight and body mass index in treatment naïve adults with HIV-1. In: 17th Annual European AIDS Clinical Society (EACS); November 6-9, 2019; Basel, Switzerland.

8. Viread [package insert]. Foster City, CA: Gilead Sciences, Inc.; 2019.

9. Molina JM, Yazdanpanah Y, Saud AA, et al. Islatravir efficacy and safety for selected demographic and baseline subgroups from a Phase 2 trial in treatment naïve adults with HIV-1 infection. In: 17th Annual European AIDS Clinical Society (EACS); November 6-9, 2019; Basel, Switzerland.

10. Molina JM, Squires K, Sax PE, et al. Doravirine versus ritonavir-boosted darunavir in antiretroviral-naive adults with HIV-1 (DRIVE-FORWARD): 96-week results of a randomised, double-blind, non-inferiority, phase 3 trial. Lancet HIV. 2020;7(1): e16-e26. doi:10.1016/S2352-3018(19)30336-4
11. Molina J-M, Squires K, Sax PE, et al. Doravirine versus ritonavir-boosted darunavir in antiretroviral-naive adults with HIV-1 (DRIVE-FORWARD): 48-week results of a randomised, double-blind, phase 3, non-inferiority trial. Lancet HIV. 2018;5(5): e211-e220. doi:10.1016/S2352-3018(18)30021-3

12. Gatell JM, Morales-Ramirez JO, Hagins DP, et al. Doravirine dose selection and 96-week safety and efficacy versus efavirenz in antiretroviral therapy-naive adults with HIV-1 infection in a phase IIb trial. Antivir Ther. 2019;24(6):425-435. doi:10.3851/IMP3323

13. Gatell JM, Raffi F, Plettenberg A, et al. Doravirine 100mg QD vs efavirenz +TDF/FTC in ART-naive HIV+ patients: week 48 results. In: CROI; February 22-25, 2016; Boston, MA, USA.

14. Orkin C, Squires K, Molina JM, et al. LB1. Doravirine/lamivudine/ tenofovir DF continues to be noninferior to efavirenz/emtricitabine/ tenofovir DF in treatment-naïve adults with HIV-1 infection: week 96 results of the DRIVE-AHEAD trial. Open Forum Infect Dis. 2018;5: S759. doi:10.1093/ofid/ofy 229.2175

15. Orkin C, Squires KE, Molina JM, et al. Doravirine/Lamivudine/ Tenofovir disoproxil fumarate is non-inferior to efavirenz/emtricitabine/tenofovir disoproxil fumarate in treatment-naive adults with human immunodeficiency virus-1 infection: week 48 results of the DRIVE-AHEAD trial. Clin Infect Dis. 2019;68(4):535-544. doi:10. $1093 / \mathrm{cid} / \mathrm{ciy} 540$

16. Wong A, Goldstein D, Mallolas J, et al. Safety and efficacy of doravirine/lamivudine/tenofovir disoproxil fumarate (DOR/3TC/ TDF) in treatment-naïve HIV-1 infected adults with transmitted NNRTI resistance mutations. J Int AIDS Soc. 2018;21:46.

17. Johnson M, Kumar P, Molina JM, et al. Switching to Doravirine/ Lamivudine/Tenofovir Disoproxil Fumarate (DOR/3TC/TDF) maintains HIV-1 virologic suppression through 48 weeks: results of the DRIVE-SHIFT trial. J Acquir Immune Defic Syndr. 2019;81(4): 463-472. doi:10.1097/QAI.0000000000002056

18. Soulie C, Santoro MM, Charpentier C, et al. Rare occurrence of doravirine resistance-associated mutations in HIV-1-infected treatment-naive patients. J Antimicrob Chemother. 2019;74(3):61 4-617. doi:10.1093/jac/dky464

19. Soulie C, Santoro MM, Storto A, et al. Prevalence of doravirine-associated resistance mutations in HIV-1-infected antiretroviral-experienced patients from two large databases in France and Italy. J Antimicrob Chemother. 2020;75(4):1026-1030. doi:10.1093/jac/dkz553

20. Guerrero-Beltrán C, Martínez-Sanz J, Álvarez M, et al. The algorithm used for the interpretation of doravirine transmitted drug resistance strongly influences clinical practice and guideline recommendations. $J$ Antimicrob Chemother. 2020;pii:dkaa009.

21. Sterrantino G, Borghi V, Callegaro AP, et al. Prevalence of predicted resistance to doravirine in HIV-1-positive patients after exposure to non-nucleoside reverse transcriptase inhibitors. Int $J$ Antimicrob Agents. 2019;53(4):515-519. doi:10.1016/j.ijantimicag.2019.02.007

22. Ayitewala A, Kyeyune F, Ainembabazi P, et al. Comparison of HIV drug resistance profiles across HIV-1 subtypes A and D for patients receiving a tenofovir-based and zidovudine-based first line regimens in Uganda. AIDS Res and Ther. 2020;17(1). doi:10.1186/s12981-0200258-7

23. Boyle A, Moss CE, Marzolini C, et al. Clinical Pharmacodynamics, Pharmacokinetics, and Drug Interaction Profile of Doravirine. Clin Pharmacokinet. 2019;58(12):1553-1565. doi:10.1007/s40262-01900806-9

24. Yee KL, DiBenedetto A, Fan L, et al. Comparative Bioavailability of Oral Granule Formulations of the HIV Antiretroviral Drugs Doravirine, Lamivudine, and Tenofovir Disoproxil Fumarate. AAPS PharmSciTech. 2020;21(3). doi:10.1208/s12249-020-1630-6

25. Wang X, Milinkovic A, Pereira B, et al. Pharmacokinetics of once-daily doravirine over $72 \mathrm{~h}$ following drug cessation. J Antimicrob Chemother. 2020;pii:dkaa038. 
26. McComsey GA, Molina JM, Yazdanpanah Y, et al. Islatravir metabolic outcomes in phase IIB trial of treatment-naive adults with HIV-1. In: CROI; March 8-11, 2020. Boston, MA, USA.

27. Rudd DJ, Cao Y, Vaddady $P$, et al. Modeling-supported islatravir dose selection for phase III. In: CROI; March 8-11, 2020; Boston, MA, USA.

28. Wensing AM, Calvez V, Ceccherini-Silberstein F, et al. 2019 update of the drug resistance mutations in HIV-1. Top Antivir Med. 2019;27 (3):111-121

29. Delstrigo [package insert]. Whitehouse Station, NJ: Merck \& CO., Inc; 2018.

30. El Zein S, Tabaja H, Kanj A, et al. Alopecia After Switch to Tenofovir Alafenamide in 6 African American Women. Open Forum Infect Dis. 2019;6(7):ofz278. doi:10.1093/ofid/ofz278

31. Khalilieh S, Yee KL, Sanchez RI, et al. Co-administration of doravirine with an aluminum/magnesium-containing antacid or pantoprazole, a proton-pump inhibitor, does not have a clinically meaningful effect on doravirine pharmacokinetics. In: IAS Conference on HIV Science; July 23-26, 2017; Paris, France.
32. Bader M, Yi Y. Gastroesophageal reflux disease in HIV-infected adults: prevalence and risk factors of moderate-severe or frequent symptoms. Open Forum Infect Dis. 2016;3(S1):1529. doi:10.1093/ ofid/ofw172.1231

33. James CW, Szabo S, Kahal D, et al. The effect of multivitamins and polyvalent cations on virologic suppression with integrase strand transfer inhibitors. AIDS. 2020;34(3):487-489. doi:10.1097/ QAD.0000000000002438

34. Yee KL, Khalilieh SG, Sanchez RI, et al. The effect of single and multiple doses of rifampin on the pharmacokinetics of doravirine in healthy subjects. Clin Drug Investig. 2017;37(7):659-667. doi:10.1007/s40261-017-0513-4

35. Khalilieh SG, Yee KL, Sanchez RI, et al. Multiple doses of rifabutin reduce exposure of doravirine in healthy subjects. J Clin Pharmacol. 2018;58(8):1044-1052. doi:10.1002/jcph.1103

36. Moher D, Liberati A, Tetzlaff J, Altman DG. The PRISMA Group (2009). Preferred Reporting Items for Systematic Reviews and MetaAnalyses: The PRISMA Statement. PLoS Med. 2009;6(7): e1000097.

\section{Publish your work in this journal}

HIV/AIDS - Research and Palliative Care is an international, peerreviewed open-access journal focusing on advances in research in HIV, its clinical progression and management options including antiviral treatment, palliative care and public healthcare policies to control viral spread. The manuscript management system is completely online and includes a very quick and fair peer-review system, which is all easy to use. Visit http://www.dovepress.com/testimonials.php to read real quotes from published authors. 